University of Nebraska - Lincoln

DigitalCommons@University of Nebraska - Lincoln

2008

\title{
Management Recommendations Based on Matrix Projection Models: The Importance of Considering Biological Limits
}

Joan Lubben

University of Nebraska-Lincoln, jolubben@dwu.edu

Brigitte Tenhumberg

University of Nebraska - Lincoln, btenhumberg2@unl.edu

Andrew J. Tyre

University of Nebraska at Lincoln, atyre2@unl.edu

Richard Rebarber

University of Nebraska - Lincoln, rrebarber1@unl.edu

Follow this and additional works at: https://digitalcommons.unl.edu/bioscifacpub

Part of the Life Sciences Commons

Lubben, Joan; Tenhumberg, Brigitte; Tyre, Andrew J.; and Rebarber, Richard, "Management Recommendations Based on Matrix Projection Models: The Importance of Considering Biological Limits" (2008). Faculty Publications in the Biological Sciences. 102.

https://digitalcommons.unl.edu/bioscifacpub/102

This Article is brought to you for free and open access by the Papers in the Biological Sciences at DigitalCommons@University of Nebraska - Lincoln. It has been accepted for inclusion in Faculty Publications in the Biological Sciences by an authorized administrator of DigitalCommons@University of Nebraska - Lincoln. 
Published in Biological Conservation 141:2 (February 2008), pp. 517-523; doi: 10.1016/j.biocon.2007.11.003

Copyright (c) 2007 Elsevier Ltd. Used by permission.

Submitted July 10, 2007; revised November 9, 2007; accepted November 14, 2007; published online January 4, 2008.

\title{
Management Recommendations Based on Matrix Projection Models: The Importance of Considering Biological Limits
}

\author{
Joan Lubben, ${ }^{1}$ Brigitte Tenhumberg, ${ }^{2}$ Andrew Tyre, ${ }^{3}$ and Richard Rebarber ${ }^{1}$ \\ 1. Department of Mathematics, University of Nebraska-Lincoln, 203 Avery Hall, Lincoln, NE 68588-0130, USA \\ 2. School of Biological Sciences, University of Nebraska-Lincoln, 348 Manter Hall, Lincoln, NE 68588-0118, USA \\ 3. School of Natural Resources, University of Nebraska-Lincoln, 416 Hardin Hall, East Campus, Lincoln, NE 68583-0974, USA \\ Corresponding author - B. Tenhumberg, tel 402 472-0267, fax 402 472-2083, email btenhumberg2@unl.edu
}

\begin{abstract}
Matrix population models are a common tool for evaluating different management strategies. In general, under deterministic analyses, management strategies are recommended that improve those matrix transitions that are most sensitive or elastic with respect to the asymptotic population growth rate, $\lambda$. These recommendations usually ignore the biological limit for these transitions. In this paper we use the endangered Serengeti cheetah (Acinonyx jubatus) as a case study to illustrate that ignoring biological limits leads to a recommendation that will not always achieve the desired goal of an asymptotic population growth rate, $\lambda \geq 1$.

We estimate the survivorships of adult cheetahs in captivity using cheetah studbook data, which is a conservative estimate of the biological limit for the adult survivorship of wild cheetahs. Our analysis suggests that adult survival sharply decreases after 8.5 years. In addition, captive cheetahs older than 18 years do not reproduce. We modify a previously published population projection matrix to include the effect of senescence on survival and fecundity. Our model suggests that increasing adult survivorship alone is not sufficient to reverse population decline. However, an additional small increase in newborn survival is likely to result in a viable cheetah population. We show these conclusions hold even in the presence of relatively large parameter perturbations.
\end{abstract}

Keywords: population projection matrix, endangered species, population management, cheetah, senescence, longevity

\section{Introduction}

Matrix projection models are a powerful tool for population management (e.g. Caswell, 2001; Morris and Doak, 2002; Hansen, 2007). In particular, the predicted long term population growth rate, $\lambda_{\text {max }}$ is an important indicator of population viability, since if $\lambda_{\max }<1$ the population decreases and eventually goes extinct. Elasticity and sensitivity analyses are the prevailing perturbation analysis; see the special feature in Ecology, Vol. 81 (3), 2000. This ap- proach evaluates the effect of infinitesimal perturbations of single parameters on $\lambda$; extrapolation to the effect of large perturbations has been criticized because the response of $\lambda_{\max }$ to changing parameter values can be nonlinear (Mills et al., 1999; Hodgson and Townley, 2004; Tenhumberg et al., in press). Perturbation analysis is also used to decide which stage or age group of a population should be manipulated to improve population viability the most (Ehrlén and Van Groenendael, 1998; De Kroon et al., 2000). Sometimes management recommendations are made solely from 
the sensitivity and elasticity analysis without evaluating whether the particular management action is sufficient to achieve the management goal (Crowder et al., 1994; Doak et al., 1994; Ratsirarson et al., 1996; Crooks et al., 1998). For example, biological limits may constrain how much life history parameters can be manipulated (Caswell, 2001). Using the endangered cheetah (Acinonyx jubatus) as a case study we illustrate that ignoring biological limits can result in management recommendations that are unlikely to achieve the management goal, which in this case is population stasis or population increase $\left(\lambda_{\max }>1\right)$.

Crooks et al. (1998) constructed a population matrix model on Serengeti cheetahs. Their model predicts an annual population decline of $8.7 \%$. The long-term population growth rate is most sensitive to adult survivorship, suggesting that wildlife managers should aim to increase adult survival. We expand the model to include the effects of senescence on survival and fecundity, which we document for captive cheetahs. We define senescence as the reduction in the rate of survival with age (Comfort, 1979; Bell, 1984; Promislow, 1991). Our model suggests that increasing adult survival alone is not sufficient to save the cheetahs, even if cheetahs live as long in the wild as in captivity. Laurenson's (1995) study suggested predation on cheetah cubs as the major factor in the survival of the cheetah while Kelly and Durant's (2000) population viability analysis showed extinction risk was highly sensitive to both adult and cub survival. Our study focuses on testing the effect of increasing adult and cub survivorships within biological limits, both individually and corporately, on the asymptotic growth rate. We use robust control methods (Hodgson and Townley, 2004; Hodgson et al., 2006; Deines et al., 2007) to evaluate the extent to which these particular life history parameters need to change in order to reach our management goal $\left(\lambda_{\max }>1\right)$.

\subsection{Natural history the cheetah (Acinonyx jubatus)}

In 1900, the cheetah (A. jubatus) roamed over much of Africa, Asia, and the Middle East with a total population estimated at 100,000 (Marker-Kraus et al., 1996). Now barely 100 years later, there are only 12,000-15,000 animals left worldwide; most reside in southern Africa (Marker-Kraus et al., 1996; Marker-Kraus, 1997; Marker-Kraus and Kraus, 1997). The largest concentration of cheetahs resides in Namibia, with an estimated population of 2500 felines (Marker-Kraus et al., 1996; Marker-Kraus, 1997; Marker-Kraus and Kraus, 1997). The majority of the cheetahs in Namibia live outside protected areas and as a result, their demise is linked to the loss of habitat by encroaching farms, poaching, and the reduced number of prey (Marker-Kraus, 1997; Marker-Kraus and Kraus, 1997; Marker et al., 2003a, 2003b). Approximately 400 cheetahs reside in the Serengeti ecosystem of Tanzania (Gros, 2002). Much of this area is a protected national park, yet the population fails to thrive due to the high cub mortality caused by predation by lions and hyenas (Eaton, 1974; Laurenson et al., 1992; Caro, 1994; Kelly et al., 1998; Kelly and Durant, 2000). Ninety-two percent of the newborn cheetahs die during the first six months of life, but once they can outrun predators (older than 5 months) their survival in- creases 10 times (Crooks et al., 1998; see also Caro, 1994). Juveniles stay with their mother for up to 18 months (Eaton, 1974; Caro, 1994; Kelly et al., 1998). A female reaches sexual maturity at approximately 24 months, and after a gestation period of about 3 months she will give birth to one to six cubs (Eaton, 1974; Caro, 1994; Kelly et al., 1998; Kelly and Durant, 2000; Marker et al., 2003b). Caro (1994) considers a 23-42 month old adult cheetah to be a young adult and those older than 42 months to just be adults. If a wild female Serengeti cheetah reaches adulthood (24 months), its average life span is 6.2 years (Kelly et al., 1998).

\section{Methods}

In this paper we extend the population projection matrix (PPM) model by Crooks et al. (1998) (see Table 1) to incorporate cheetah senescence. We maintain the original 6 month age classes as an aid to help compare models which include senescence with those that do not. The new model (see Table 2) increases the number of age classes from 8 to 36; the last age class includes cheetahs that are 210-216 month old (18 years). Since cheetahs older than 18 years have zero fecundity, they are considered "biologically dead" and are not a factor in the cheetah's population growth, hence they are ignored in the model (Caswell, 2001). We then identify strategies that produce a growing population $(\lambda>1)$ and are robust to parameter uncertainty. First, we estimate the biological limit of adult survival. Then, within the biological meaningful range of survival scenarios, we identify the magnitude of disturbances permissible to reach our management goal; if the permissible perturbations are large we call the management strategy "robust".

\subsection{Estimating maximum adult survival}

We analyze survival data available in the 2002, 2003, and 2004 International Cheetah Studbooks (Marker, 2004; Marker, 2006a; Marker, 2006b). Because the model by Crooks et al. (1998) ignores males, we only estimate female survival. Sometimes cheetah deaths are not reported and as a result the records suggest that some of these cheetahs are older than 30 years; even though in captivity cheetahs rarely live

Table 1. Population projection matrix (PPM) for wild cheetahs (Crooks et al., 1998)

\begin{tabular}{lccccccc}
\hline \multicolumn{7}{l}{ Age classes (months) } \\
\hline $0-6$ & $6-12$ & $12-18$ & $18-24$ & $24-30$ & $30-36$ & $36-42$ & $42+$ \\
\hline 0 & 0 & 0 & 0 & $s_{2} f_{1}$ & $s_{2} f_{1}$ & $s_{2} f_{1}$ & $s_{3} f_{2}$ \\
$s_{0}$ & 0 & 0 & 0 & 0 & 0 & 0 & 0 \\
0 & $s_{1}$ & 0 & 0 & 0 & 0 & 0 & 0 \\
0 & 0 & $s_{1}$ & 0 & 0 & 0 & 0 & 0 \\
0 & 0 & 0 & $s_{2}$ & 0 & 0 & 0 & 0 \\
0 & 0 & 0 & 0 & $s_{2}$ & 0 & 0 & 0 \\
0 & 0 & 0 & 0 & 0 & $s_{2}$ & 0 & 0 \\
0 & 0 & 0 & 0 & 0 & 0 & $s_{2}$ & $s_{3}$ \\
\hline
\end{tabular}

Values used in PPM: $s_{0}=0.081$, survival of $0-6$ month age class; $s_{1}=0.771$, survival of 6-12 and 12-18 month age classes; $s_{2}=0.920$, survival of 18-24, 24-30, 30-36 and 36-42 month age classes; $s_{3}=0.879$, survival of $42+$ month age class; $f_{1}=1.2476$, fecundity of $24-30,30-36$, and $36-42$ month age classes; $f_{2}=1.4994$, fecundity of $42+$ month age class. 
Table 2. Population projection matrix (PPM) for wild cheetahs incorporating senescence

\begin{tabular}{|c|c|c|c|c|c|c|c|c|c|c|c|c|c|c|c|c|}
\hline \multicolumn{17}{|c|}{ Age classes (months) } \\
\hline $0-6$ & $6-12$ & $12-18$ & $18-24$ & $24-30$ & $30-36$ & $36-42$ & $42-48$ & $\ldots$ & 96-102 & 102-108 & $\ldots$ & 138-144 & $144-150$ & $\ldots$ & 204-210 & $210-216$ \\
\hline 0 & 0 & 0 & 0 & $s_{2}^{*} f_{1}$ & $s_{2}^{*} f_{1}$ & $s_{2}^{*} f_{1}$ & $s_{3}^{*} f_{2}$ & $\ldots$ & $s_{3}^{*} f_{2}$ & $s_{4}^{*} f_{2}$ & $\ldots$ & $s_{4}^{*} f_{2}$ & $s_{4}^{*} f_{2}$ & $\ldots$ & $s_{4}^{*} f_{3}$ & 0 \\
\hline 0 & $s_{1}$ & 0 & 0 & 0 & 0 & 0 & 0 & $\ldots$ & 0 & 0 & $\ldots$ & 0 & 0 & $\ldots$ & 0 & 0 \\
\hline 0 & 0 & $s_{1}$ & 0 & 0 & 0 & 0 & 0 & $\ldots$ & 0 & 0 & $\ldots$ & 0 & 0 & $\ldots$ & 0 & 0 \\
\hline 0 & 0 & 0 & $s_{2}^{*}$ & 0 & 0 & 0 & 0 & $\ldots$ & 0 & 0 & $\ldots$ & 0 & 0 & $\ldots$ & 0 & 0 \\
\hline 0 & 0 & 0 & 0 & 0 & 0 & 0 & $s_{3}^{*}$ & $\cdots$ & 0 & 0 & $\ldots$ & 0 & 0 & $\ldots$ & 0 & 0 \\
\hline$\ldots$ & $\ldots$ & $\ldots$ & $\ldots$ & $\ldots$ & $\ldots$ & $\ldots$ & $\ldots$ & $\ldots$ & $\ldots$ & $\ldots$ & $\ldots$ & $\ldots$ & $\ldots$ & $\ldots$ & $\ldots$ & $\ldots$ \\
\hline 0 & 0 & 0 & 0 & 0 & 0 & 0 & 0 & $\ldots$ & $s_{3}^{*}$ & 0 & $\ldots$ & 0 & 0 & $\ldots$ & 0 & 0 \\
\hline 0 & 0 & 0 & 0 & 0 & 0 & 0 & 0 & $\ldots$ & $0^{\circ}$ & $S_{4}^{*}$ & $\ldots$ & 0 & 0 & $\ldots$ & 0 & 0 \\
\hline$\ldots$ & $\ldots$ & $\ldots$ & $\ldots$ & $\ldots$ & $\ldots$ & $\ldots$ & $\ldots$ & $\ldots$ & $\ldots$ & $\ldots$ & $\ldots$ & $\ldots$ & $\ldots$ & $\ldots$ & $\ldots$ & $\ldots$ \\
\hline 0 & 0 & 0 & 0 & 0 & 0 & 0 & 0 & $\ldots$ & 0 & 0 & $\ldots$ & $S^{*}{ }_{4}$ & 0 & $\ldots$ & 0 & 0 \\
\hline
\end{tabular}

Values used in PPM: $s_{0}=0.081$, survival of 0-6 month age class; $s_{1}=0.771$, survival of $6-12$ and $12-18$ month age classes; $s^{*}=0.9685$, survival of $18-24, \ldots, 36-42$ month age classes; $s_{3}^{*}=0.9567$, survival of $42-48, \ldots, 96-102$ month age classes; $s_{4}^{*}=0.8980$, survival of $102+$ month age classes; $f_{1}=1.2476$, fecundity of 24-30, 30-36, and 36-42 month age classes; $f_{2}=1.4994$, fecundity of $42-144$ month age classes; $f_{3}=1.4994$, fecundity of $144-$ $150, \ldots, 210-216$ month age classes.

past 21 years of age (Marker-Kraus, 1997). Also, since the inclusion of live cheetahs did not change the survivorship estimation significantly, in the survival analysis we exclude those cheetahs that are still alive and those considered "lost" or "assumed dead" by the stud keeper. This left $N=1955$ cheetahs for our analysis. Figure 1 shows the Kaplan-Meier estimates of cheetah survival (Cox and Oakes, 1984). Analogous to Crooks et al. (1998) model, we use 6 month time intervals and calculate survivorships for 0-6 month old $\left(s_{0}^{*}\right), 6-18$ months old $\left(s^{*}\right)$, and 18-42 months old cheetahs $\left(s_{2}^{*}\right)$. To account for senescence (Figure 1) we split the survival of the $42+$ month old cheetahs of Crooks et al. (1998) model into 42-102 months old $\left(s_{3}^{*}\right)$ and $>102$ months old $\left(s^{*}{ }_{4}\right)$. For cheetahs older than 156 months we use the same survivorship as for cheetahs aged 102-156 months, even though this is an overestimation. We calculate the average survival per time step as follows:

$s_{i}^{*}=\left(\frac{\ell(t+\Delta t)}{\ell(t)}\right)^{1 / n}$

where $\ell$ is the Kaplan-Meier estimate, $t$ and $(t+\Delta t)$ are the start and end of $s^{*}$, respectively, and $n$ is the number of time steps in $\Delta t$ (Caswell, 2001). Using the estimates for survivorship listed in Table 3, our model predicts that once a cheetah survives to adulthood, there is a $50 \%$ chance that she will survive past 9-91/2 years (108-114 months).

\subsection{Estimating fecundity}

The model by Crooks et al. (1998) assumes the same fecundity, $f_{2}$, for all $42+$ months old cheetahs. This is particularly problematic because in the wild, cheetahs do not reproduce after 12 years of age (Nowell and Jackson, 1996; Kelly and Durant, 2000; Marker et al., 2003b) and even in captivity, cheetahs rarely, if at all, reproduce after they turn
18 years (216 months) (see Marker-Kraus, 1997; Marker, 2000, 2004, 2006a, 2006b). In captivity, a cheetah's prime reproductive period is between 3 and 10 years of age (Marker, 2000, 2004, 2006a, 2006b), but in general cheetah fecundity in captivity is lower than in the wild (Nowell and Jackson, 1996; also compare Marker (2000, 2004, 2006a, 2006b) with Kelly and Durant (2000). This is in agreement with other captive carnivorous animals (Laike, 1999; Clubb and Mason, 2007). Because we do not have data to estimate the effect of senescence on the fecundity of wild cheetahs, we consider two different cases. In both cases, we assume fecundity of the 18-42 month old cheetahs $\left(f_{1}=1.2476\right)$ and the $42-144$ month old cheetahs $\left(f_{2}=1.4994\right)$ is that given in Crooks et al. (1998). Defining $f_{3}$ to be the fecundity of the 144-216 month old cheetahs, we first set $f_{3}$ equal to $f_{2}$ $\left(f_{3}=1.4994\right)$, which ignores a reduction in fecundity due to senescence, and as a consequence, our model's predictions will overestimate population growth rate (Table 2). Second, we assume that adult cheetahs stop reproducing at the age of 12 years as is observed in wild cheetahs, $\left(f_{3}=0\right)$, in which case we can remove these age classes from the model. In both cases, we assume the fecundity of cheetahs older than 216 months (18 years) is zero.

\subsection{Perturbation analysis}

In this analysis we examine the response of the asymptotic population growth, $\lambda_{\max }$ to large simultaneous deviations from the parameter values used in the model by Crooks et al. (1998); in particular we wish to explore if it is theoretically possible to achieve our management goal (in this paper $\lambda_{\max }>1$ ) by perturbing parameter values to their biological limits. First we set the fecundities and survivorships of the 102-216 month old cheetahs to their biological limit $\left(f_{2}=f_{2}=1.4994, s^{*}{ }^{\prime}\right.$, Table 2$)$. We then identify the survivorship combinations of younger adult cheetahs $\left(s^{*}, s_{3}^{*}\right)$ 


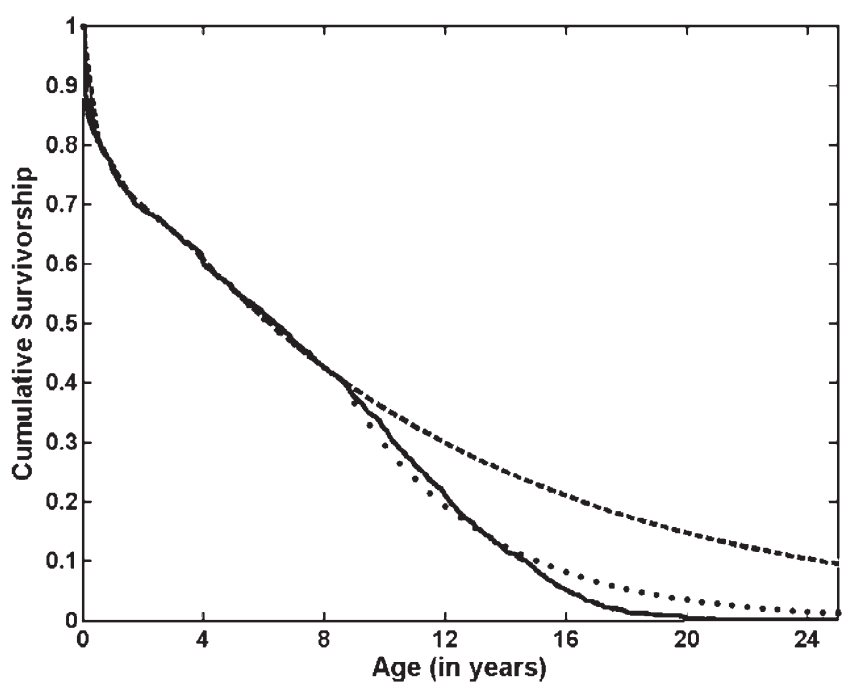

Figure 1. Survival data from captive female cheetahs shows the cumulative survivorship of female cheetahs with known age of death. The solid curve is the Kaplan-Meier curve using monthly time steps. The heavy dashed curve shows the cumulative female adult survivorship with all $42+$ month old cheetahs lumped into one age class with the survivorship of 42-102 month cheetahs $\left(s_{3}^{*}\right)$. The heavy dotted curve divides the $42+$ month old cheetahs into two age classes: $42-102$ months $\left(s_{3}^{*}\right)$ and 102+ months $\left(s_{4}^{*}\right)$ with the $102+$ month age class having the survivorship of 102162 month cheetahs. The 95\% confidence intervals for the KaplanMeier curve are very tight and thus are omitted for clarity.

that yield a growth rate of $\lambda=1$. Let $A$ be the $36 \times 36$ population projection matrix model shown in Table 2 with Crooks et al. (1998) values for the survivorships of the 0-6 month cubs $\left(s_{0}\right)$ and the 6-18 month old cheetahs $\left(s_{1}\right)$. Let $\boldsymbol{P}$ denote the 36 by 36 matrix containing the deviations from the nominal values given in $A$; all matrix entries of $\boldsymbol{P}$ that are not affected by the particular perturbation are zero. Since we are perturbing $s_{2}^{*}$ and $s_{3}^{*}$, we let $p_{2}$ denote changes in $s_{2}^{*}$, and we let $p_{3}$ denote changes in $s_{3}^{*}$. Thus the first row of the matrix $\boldsymbol{P}$ has $p_{2} f_{1}$ in columns 5 through 7 and $p_{3} f_{2}$ in columns 8 through 17 where $f_{1}$ and $f_{2}$ are the fecundities of the 18-42 month old and the 42-216 month old cheetahs. The subdiagonal of $\boldsymbol{P}$ has $p_{2}$ in columns 4 through 7 and $p_{3}$ in columns 8 through 17. We call $\boldsymbol{A}+\boldsymbol{P}$ the perturbed matrix. For example, to examine the effect of changing both $s^{*}{ }_{2}, s_{3}^{*}$ to that given in Crooks et al. (1998) we set $p_{2}=-.0485$ and $p_{3}=-.0468$ in the above $\boldsymbol{P}$. This gives $s_{2}^{*}+$ $p_{2}=s_{2}$ and $s_{3}^{*}+p_{3}=s_{3}$.

The asymptotic population growth rate of the perturbed matrix $A+P$ is the largest eigenvalue, $\lambda$, of $A+P$ (Caswell, 2001). The eigenvalues of our reducible $36 \times 36$ matrix are the same as the eigenvalues of the primitive $35 \times 35$ submatrix plus the eigenvalue equal to 0 ( $\mathrm{O}^{\prime} \mathrm{Nan}, 1976$ : p. 71). Since $\lambda$ depends upon the choice of parameters $p_{2}$ and $p_{3}$, we denote it by $\lambda\left(p_{2}, p_{3}\right)$. Our goal is to determine which $\left(p_{2}, p_{3}\right)$ yield $\lambda\left(p_{2}, p_{3}\right) \geq 1$. The curve $\lambda\left(p_{2}, p_{3}\right)=1$ in the $\left(p_{2}, p_{3}\right)$ plane can be found by solving the equation $\operatorname{det}(I-$ $(\boldsymbol{A}+\boldsymbol{P}))=0$ where $\boldsymbol{I}$ is the identity matrix of appropriate size. Deines et al. (2007) proved the $\lambda\left(p_{2}, p_{3}\right)=1$ curve divides the $\left(p_{2}, p_{3}\right)$ plane into increasing $\left(\lambda\left(p_{2}, p_{3}\right)>1\right)$ and decreasing $\left(\lambda\left(p_{2}, p_{3}\right)<1\right)$ populations. The general results in Boeckner et al. (in preparation) can be applied to show that if $\lambda\left(p_{2}, p_{3}\right)=1$, then $\lambda\left(p_{2}, p_{3}\right)=1$ is the largest eigenvalue. Methods for solving the equation $\operatorname{det}(I-(A+P))=0$ are further outlined in Hodgson and Townley (2004) and Hodgson et al. (2006) and in Deines et al. (2007). Because cub survival in the wild is extremely low and can theoretically be increased to a large extent we also consider the perturbations in three parameter values: $s_{0}, s_{2}^{*}$, and $s_{3}^{*}$.

\section{Results}

In this paper we used captive cheetahs as a conservative estimate of the biological limit of adult survival. Figure 1 illustrates that the survival of captive cheetahs dropped after 8.5 years and that a model ignoring this largely overestimates cheetah survival (heavy dashed line). Thus, predicting the effect of fundamentally increasing adult survival requires a model that includes a senescent age class (modified model Table 2, and heavy dotted line in Figure 1). Assuming that the survival of an adult cheetah in the wild can be raised to that in captivity corresponds to an average life expectancy of 9-91/2 years for those cheetahs that reach adulthood. This is four years more than in the model by Crooks et al. (1998) and three years longer than calculated by Kelly et al. (1998). Still, this increase in adult survival is not sufficient to produce a growing population; the projected population growth rate, $\lambda$, is 0.998 , indicating that each 6 month interval the cheetah population decreases by $0.2 \%$ (Table 2 ).

Next we perturbed the survival of 18-42 months old $\left(s_{2}\right)$ and 42-102 months old cheetahs $\left(s_{3}\right)$ (Figure 2). Only if both survival parameters increase to that of captive cheetahs, and cheetah fecundity is not affected by senescence (e.g. fecundity of 12-18 year old females does not differ from 4 year old ones) the projected population growth rate is close to achieving population stasis as shown by the short distance to $\lambda=1$ curve. In a more realistic scenario, where females older than 12 years of age are assumed to be infertile, the distance to the $\lambda=1$ curve is rather large. Hence within

Table 3. Survivorship of captive and wild female cheetahs (see Figure 1)

\begin{tabular}{|c|c|c|c|}
\hline \multicolumn{2}{|l|}{ Age classes in months } & \multirow{2}{*}{$\begin{array}{l}\text { Survivorship captivity }^{\mathrm{a}} \\
S_{0}^{*}=0.8087 \pm 0.0174\end{array}$} & \multirow{2}{*}{$\begin{array}{c}\text { Survivorship Crooks et al. (1998) } \\
\qquad s_{0}=0.081\end{array}$} \\
\hline $0-6$ & Small cubs & & \\
\hline $6-12,12-18$ & Medium and weaned cubs & $s^{*}=0.9434 \pm 0.082$ & $s_{1}=0.771$ \\
\hline $18-24,24-30,30-36$, and $36-42$ & Young adults & $s_{2}^{*}=0.9685 \pm 0.047$ & $s_{2}=0.920$ \\
\hline $42-48,48-56, \ldots 96-102$ & Adults & $s_{3}^{*}=0.9567 \pm 0.0041$ & \\
\hline $102+$ & Old adults & $s_{4}^{*}=0.8980 \pm 0.0091$ & \\
\hline
\end{tabular}

\footnotetext{
${ }^{a}$ With $95 \%$ confidence intervals.
} 


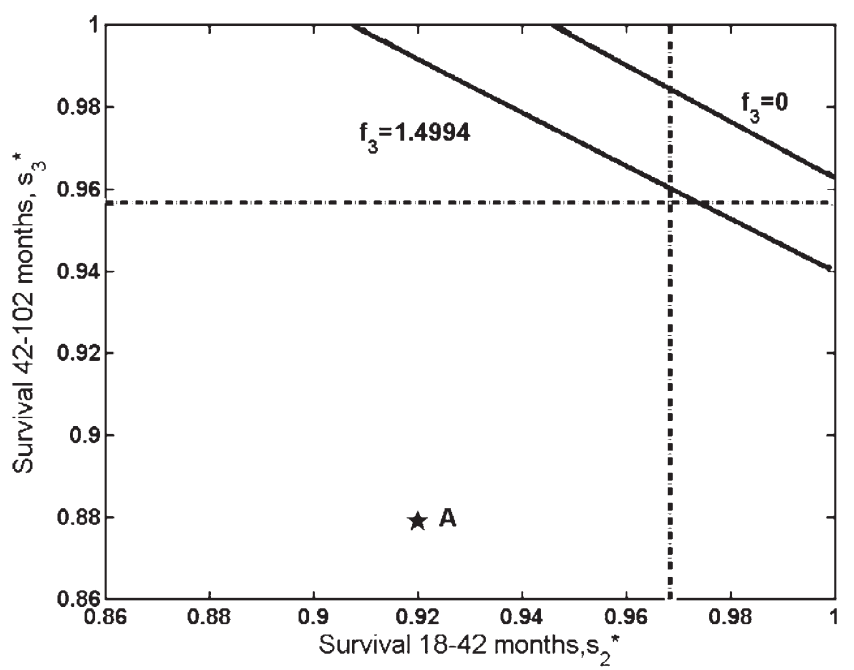

Figure 2. Perturbed survivorships of 18-42 month old adults and 42-102 month old adults where the 102+ month old adult survivorships have been set to the biological limit $\left(s_{4}^{*}=0.898\right)$. The solid curves denotes $\lambda=1$ for two different fecundities of the 144216 month old cheetahs: $f_{3}=1.4994$ and $f_{3}=0$. Points above each of these curves produce positive growth; points below the curve produce negative growth. The horizontal line denotes the biological limit for the survivorship of 42-102 months old adults $\left(s_{3}^{*}=\right.$ $0.9567)$ and the vertical line denotes the biological limit for the survivorship of $18-42$ months old adults $\left(s^{*}=0.9685\right)$. Point A marks the original, unperturbed survivorships $\left(s_{2}=0.92, s_{3}=0.879\right.$, $\lambda=0.9553$ ) reported by Crooks et al. (1998).

the biological meaningful parameter range it is highly unlikely to produce population stasis $\left(\lambda_{\max }=1\right)$ by increasing adult survival alone (Figure 2 ). It is only possible to achieve a growing population by increasing adult survival to its biological limit if the fecundity of younger adults is underestimated by Crooks et al. (1998) (Figure 3).

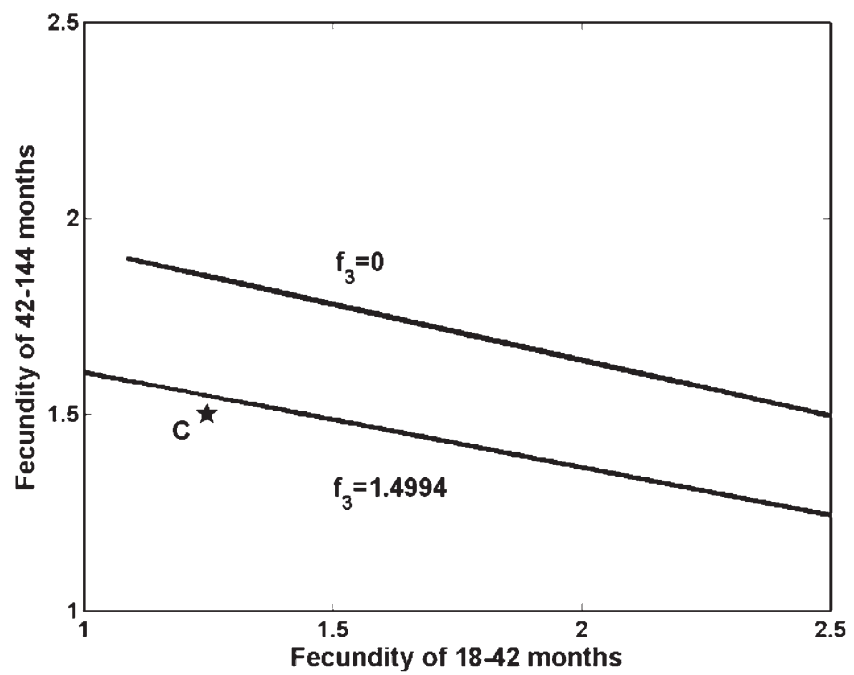

Figure 3. Necessary fecundities required to achieve $\lambda=1$ with all adult survivorships at their biological limits. The top curve sets the fecundity of $144-216$ month old to be zero $\left(f_{3}=0\right)$. The bottom curve sets the fecundities of the 144-216 month olds to be the same as the $42-102$ month old adults $\left(f_{3}=1.4994\right)$. Point $C$ shows the fecundities as calculated by Crooks et al. (1998).

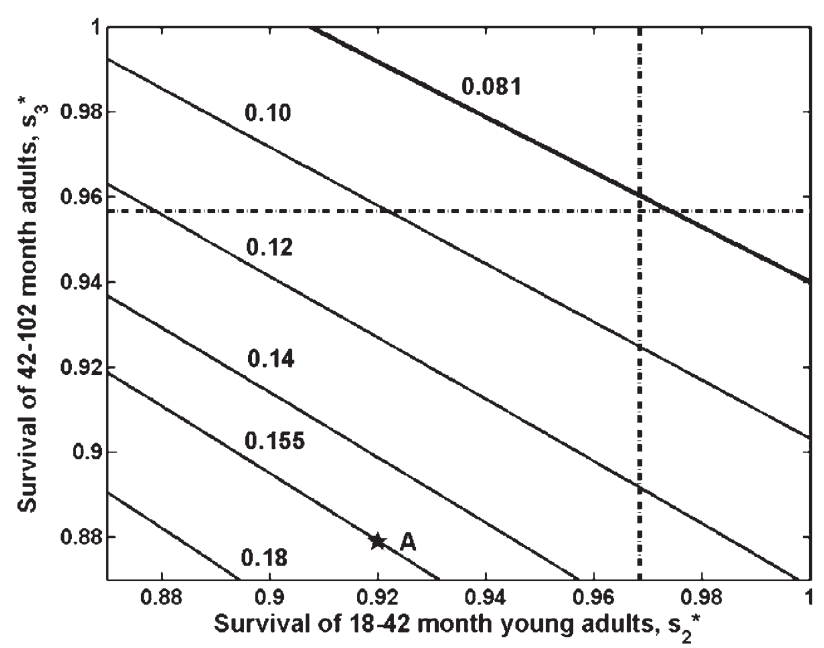

Figure 4. Contour graph of the perturbed survivorships of the 1842 month old adult, 42-102 month old adult and 0-6 month cubs. The contours are labeled by the 0-6 month cub survivorship and describe the 18-42 month and 42-102 month survivorships necessary to achieve stasis $(\lambda=1)$. The heavy contour denotes calculated 0-6 month cub survivorship $\left(s_{0}=0.081\right)$. The horizontal line denotes the biological limit for the adult survivorship of 42-102 month cheetahs $\left(s_{3}^{*}=0.9567\right)$. The vertical line denotes the biological limit in the $18-42$ month old cheetahs $\left(s_{2}^{*}=0.9685\right)$. Point A marks the original, unperturbed young and old adult survivorships as recorded by Crooks et al. (1998) $\left(s_{2}=.92, s_{3}=.879\right.$, $\lambda=0.9553)$. In this case, the $0-6$ month cub survivorship must increase to 0.155 in order to obtain $\lambda=1$.

Finally we explored the effect of increasing newborn survival (Figure 4), which is highly impacted by predators (Caro, 1994; Laurenson, 1995). Our model suggests that increasing newborn survival from 0.081 to only 0.155 , which is far below cub survival in captivity $(=0.81$, Table 3$)$, produces population stasis. The improvement in cub survival required to achieve population stasis decreases with increasing survival of older age classes.

\section{Discussion}

Our results reveal that senescence plays an important role in the longevity of the captive adult cheetah. In the wild, the evidence for senescence is ambiguous (Promislow, 1991; Gaillard et al., 1994; Slade, 1995) due to many reasons, including the fact that wild animals are subjected to predation, injuries, and random environmental factors and hence rarely live to old age (Medawar, 1952; Comfort, 1979). Furthermore there is a lack of long-term monitoring which also contributes to the inability to quantify senescence (Gaillard et al., 1994; Nichols et al., 1997; Loison et al., 1999). Thus population projection matrices for wild animals rarely incorporate senescence into the model. In captivity, senescence is exhibited by increased mortality and decreased fecundity of older animals, which cannot be explained by random effects (Comfort, 1979). Since the survival of adult Serengeti cheetahs is already very close to the biological limit, predicting the effect of increasing adult survivorship even further requires a model which takes senescence into consideration - to do otherwise will vastly 
overestimate adult survivorship and in turn, result in an overestimation of the population growth rate.

In this paper we calculated the survivorships of the adult captive cheetah and used these to approximate the biological limit for the wild cheetah. Using captive reared animals to estimate biological limits assumes that hunting success of adult cheetahs (food availability) is independent of age and that diseases successfully treated in captivity have negligible survival consequences for wild animals. Since captive cheetahs have been known to live more than 15 years (Marker-Kraus, 1997; Marker, 2004; Marker, 2006a; Marker, 2006b), husbandry methods are not a restricting factor when determining the biological limit. We also assume that cheetahs older than 18 years were infertile. Using this model, we find that even increasing the survivorships of the wild adult cheetah to the biological limit does not result in an asymptotic growing population. Since it is highly unlikely the survivorship of the wild cheetah can reach and be sustained at these biological limits, a management strategy which only strives to increase adult survivorship will not result in a growing population.

Our perturbation analysis of the model evaluated the effect of increasing the adult survivorship alone and then also in conjunction with increasing the 0-6 month cub survivorship. Since there is uncertainty in all parameter estimates where some of these uncertainties maybe large, it is important to consider management options which will succeed in light of this uncertainty. Since we are interested in the effect of changing two (or three) parameters (or the effect of the uncertainty in these values), we use robustness methods as described in Hodgson and Townley (2004) and Hodgson et al. (2006), and Deines et al. (2007). We calculate the curve $\lambda=1$; the distance from model prediction to this curve is a measure of robustness (the larger the distance, the larger the robustness).

This perturbation analysis suggests that within the biological reasonable parameter space it is highly unlikely to produce a growing population by increasing adult survival alone. In contrast, increasing newborn survival to a fraction of its biological limit can achieve our management goal. The latter management strategy is robust to parameter uncertainty. The survivorship of Serengeti cubs is currently an order of magnitude smaller than both the biological limit and that of Namibian wild cubs where predation on cubs is not an issue (Marker et al., 2003b). In the Serengeti, cheetah predators are protected from culling, therefore it will be challenging to find effective management strategies ensuring better newborn survival. In addition, it is necessary to account for the relative costs of altering different parameters (Baxter et al., 2006). However this paper clearly demonstrates that such strategies could dramatically improve the future prospects of Serengeti cheetah populations.

\section{Acknowledgments}

This work was supported by NSF REU Site Grant 0354008. Richard Rebarber was supported in part by NSF Grant 0606857.

\section{References}

Baxter et al., 2006 P. W. J. Baxter, M. A. McCarthy, H. P. Possingham, P. W. Menkhorst and N. McLean, Accounting for management costs in sensitivity analyses of matrix population models, Conservation Biology 20 (2006), pp. 893-905.

Bell, 1984 G. Bell, Evolutionary and nonevolutionary theories of senescence, The American Naturalist 124 (1984), pp. 600-603.

Boeckner et al., in preparation D. Boeckner, J. Lubben, R. Rebarber, S. Townley, B. Tenhumberg, The leading eigenvalue of population projection models (in preparation).

Caro, 1994 T. M. Caro, Cheetahs of the Serengeti Plains: Group Living of an Asocial Species, University of Chicago Press, Chicago (1994).

Caswell, 2001 H. Caswell, Matrix Population Models: Construction, Analysis and Interpretation (Second ed.), Sinauer Associates, Inc., Sunderland, Massachusetts (2001).

Clubb and Mason, $2007 \rightarrow$ R. Clubb and G. J. Mason, Natural behavioral biology as a risk factor in carnivore welfare: How analysing species differences could help zoos improve enclosures, Applied Animal Behavioral Science 102 (2007), pp. 303-328.

Comfort, 1979 A. Comfort, The Biology of Senescence (Third ed.), Springer, Berlin (1979).

Cox and Oakes, $1984 \longrightarrow$ D. R. Cox and D. Oakes, Analysis of Survival Data (Monographs on Statistics and Applied Probability), Chapman and Hall, London (1984).

Crooks et al., $1998 \rightarrow$ K. R. Crooks, M. A. Sanjayan and D. F. Doak, New insights on cheetah conservation through demographic modeling, Conservation Biology 12 (1998), pp. 889-895.

Crowder et al., $1994>$ L. B. Crowder, D. T. Crouse, S. S. Heppell and T. H. Martin, Predicting the impact of turtle excluder devices on loggerhead sea turtle populations, Ecological Applications 4 (1994), pp. 437-445.

Deines et al., 2007 A. Deines, E. Peterson, D. Boeckner, J. Boyle, A. Keighley, J. Kogut, J. Lubben, R. Rebarber, R. Ryan, B. Tenhumberg, S. Townley and A. J. Tyre, Robust population management under uncertainty for structured population models, Ecological Applications 17 (2007), pp. 2175-2183.

De Kroon et al., $2000>$ H. De Kroon, J. Van Groenendael and J. Ehrlén, Elasticities: A review of methods and model limitations, Ecology 81 (2000), pp. 607-618.

Doak et al., 1994 D. Doak, P. Kareiva and B. Klepetka, Modeling population viability for the desert tortoise in the western Mojave desert, Ecological Applications 4 (1994), pp. 446-460.

Eaton, 1974 R. L. Eaton, The Cheetah: The Biology, Ecology, and Behavior of an Endangered Species, Van Nostrand Reinhold, New York (1974).

Ehrlén and Van Groenendael, 1998 J. Ehrlén and J. Van Groenendael, Direct perturbation analysis for better conservation, Conservation Biology 12 (1998), pp. 470-474.

Gaillard et al., 1994 J.-M. Gaillard, D. Allaine, D. Pontier, N. G. Yoccoz and D. E. L. Promislow, Senescence in natural populations of mammals: A reanalysis, Evolution 48 (1994), pp. 509-516. 
Gros, $2002>$ P. M. Gros, The status and conservation of the cheetah Acinonyx jubatus in Tanzania, Biological Conservation 106 (2002), pp. 177-185.

Hansen, $2007 \rightarrow$ M. J. Hansen, Evaluating management strategies and recovery of an invasive grass (Agropyron cristatum) using matrix population models, Biological Conservation 140 (2007), pp. 91-99.

Hodgson and Townley, 2004 D. J. Hodgson and S. Townley, Linking management changes to population dynamic responses: the transfer function of a projection matrix perturbation, Journal of Applied Ecology 41 (2004), pp. 1155-1161.

Hodgson et al., 2006 D. J. Hodgson, S. Townley and D. McCarthy, Robustness: predicting the effects of life history perturbations on stage-structured population dynamics, Theoretical Population Biology 70 (2006), pp. 214-224.

Kelly et al., 1998 M. J. Kelly, M. K. Laurenson, C. D. FitzGibbon, D. A. Collins, S. M. Durant, G. W. Frame, B. C. R. Bertram and T. M. Caro, Demography of the Serengeti cheetah (Acinonyx jubatus) population: the first 25 years, Journal of Zoology, London 244 (1998), pp. 473-488.

Kelly and Durant, $2000 \rightarrow$ M. J. Kelly and S. M. Durant, Viability of the Serengeti cheetah population, Conservation Biology 14 (2000), pp. 786-797.

Laike, 1999 L. Laike, Conservation genetics of nordic carnivores: lessons from zoos, Hereditas 130 (1999), pp. 203-216.

Laurenson, 1995 M. K. Laurenson, Implications of high offspring mortality for cheetah population dynamics. In: A. R. E. Sinclair and P. Arcese, editors, Serengeti II: Dynamics, Management, and Conservation of an Ecosystem, University of Chicago Press, Chicago (1995), pp. 385-399.

Laurenson et al., 1992 M. K. Laurenson, T. Caro and M. Borner, Female cheetah reproduction, National Geographic Research and Exploration 8 (1992), pp. 64-75.

Loison et al., 1999 A. Loison, M. Festa-Bianchet, J. -M. Gaillard, J. T. Jorgenson and J.-M. Jullien, Age-specific survival in five populations on ungulates: evidence of senescence, Ecology 80 (1999), pp. 2539-2554.

Marker, 2000 L. Marker, 1999 Cheetah (Acinonyx jubatus) Studbook, Cheetah Conservation Fund, Namibia (2000).

Marker, 2004 L. Marker, 2002 Cheetah (Acinonyx jubatus) Studbook, Cheetah Conservation Fund, Namibia (2004).

Marker, 2006a L. Marker, 2003 Cheetah (Acinonyx jubatus) Studbook, Cheetah Conservation Fund, Namibia (2006).

Marker, 2006b L L. Marker, 2004 Cheetah (Acinonyx jubatus) Studbook, Cheetah Conservation Fund, Namibia (2006).

Marker et al., 2003a $>$ L. L. Marker, A. J. Dickman, M. G. L. Mills and D. W. Macdonald, Aspects of the management of cheetahs Acinonyx jubatus jubatas trapped on Namibian farmlands, Biological Conservation 114 (2003), pp. 401-412.
Marker et al., 2003b L. L. Marker, A. J. Dickman, R. M. Jeo, M. G. L. Mills and D. W. Macdonald, Demography of the Namibian cheetah, Acinonyx jubatus jubatas, Biological Conservation 114 (2003), pp. 413-425.

Marker-Kraus, 1997 L. Marker-Kraus, History of the cheetah Acinonyx jubatus in zoos 1829-1994, International Zoo Yearbook 35 (1997), pp. 27-43.

Marker-Kraus et al., 1996 L. Marker-Kraus, D. Kraus, D. Barnett and S. Hurlbut, Cheetah Survival on Namibian Farmlands, Cheetah Conservation Fund, Windhoek (1996).

Marker-Kraus and Kraus, 1997 Marker-Kraus, L., Kraus, D., 1997. Conservation strategies for the long-term survival of the cheetah Acinonyx jubatus by the Cheetah Conservation Fund, Windhoek. International Zoo Yearbook 35, 59-66.

Medawar, 1952 P. B. Medawar, An Unsolved Problem in Biology, H. K. Lewis, London (1952).

Mills et al., 1999 L. S. Mills, D. F. Doak and M. J. Wisdom, Reliability of Conservation Actions Based on Elasticity Analysis of Matrix Models, Conservation Biology 13 (1999), pp. 815-829.

Morris and Doak, $2002 \longrightarrow$ W. F. Morris and D. F. Doak, Quantitative Conservation Biology; Theory and Practice in Conservation Biology, Sinauer Associates, Sunderland, Massachusetts (2002).

Nichols et al., 1997 J. D. Nichols, J. E. Hines and P. Blums, Tests for senescent decline in annual survival probabilities of common pochards, Aythya ferina, Ecology 78 (1997), pp. 1009-1018.

Nowell and Jackson, 1996 K. Nowell and P. Jackson, editors, Wild Cats, Status Survey and Conservation Action Plan, IUCN, Gland, Switzerland (1996).

O'Nan, 1976 M. O'Nan, Linear Algebra (second ed.), Harcourt Brace Jovanovich, Inc., New York (1976).

Promislow, 1991 D. E. L. Promislow, Senescence in natural populations of mammals: a comparative study, Evolution 45 (1991), pp. 1869-1887.

Ratsirarson et al., 1996 J. Ratsirarson, J. A. Silander Jr. and A. F. Richard, Conservation and management of a threatened Madagascar palm species, Neodypsis decaryi, Jumelle, Conservation Biology 10 (1996), pp. 40-52.

Slade, $1995-$ N. A. Slade, Failure to detect senescence in persistence of some grassland rodents, Ecology 76 (1995), pp. 863-870.

Tenhumberg et al., in press B. Tenhumberg, S. Louda, J. Eckberg, M. Takahashi, Monte Carlo analysis of parameter uncertainty in matrix models of the weed Cirsium vulgare. Journal of Applied Ecology (in press). 